\title{
APORTES AL DEBATE SOBRE EL ABORTO EN CHILE: DERECHOS, GÉNERO Y BIOÉTICA
}

\section{Claudia Dides C.*}

Resumen: El presente artículo es un ensayo reflexivo sobre el problema del aborto en Chile, a partir de la revisión del contexto histórico actual del país, así como un planteamiento crítico desde la perspectiva de género y bioética. El análisis se ha organizado en tres apartados: el contexto del aborto en Chile en el marco de los derechos humanos, las transformaciones en la intimidad y los aportes de la bioética y la perspectiva de género al debate. Por último, se presentan, a modo de reflexión, algunas de las tensiones existentes así como las interrogantes para posibles investigaciones.

Palabras clave: aborto, debate, derechos humanos, derechos sexuales y reproductivos, género, bioética

\section{CONTRIBUTIONS TO THE DEBATE ABOUT ABORTION IN CHILE: RIGHTS, GENDER AND BIOETHICS}

Abstract: This paper is a reflexive essay about the abortion problem in Chile, considering the current historical context as well as a critical approach from the gender and bioethical perspective. The analysis has been organized in three tracks: the abortion context in Chile in relation to the human rights, the changes in privacy and the contribution of bioethics and gender perspective to this debate. Finally, for reflection, some current tensions as well as questions for future studies are presented.

Key words: abortion, debate, human rights, sexual and reproductive rights, gender, bioethics

\section{CONTRIBUIÇÕES AO DEBATE SOBRE O ABORTO NO CHILE: DIREITOS, GÊNERO E BIOÉTICA}

Resumo: O presente artigo é um ensaio reflexivo sobre o problema do aborto no Chile, a partir da revisão do contexto histórico atual do país, assim como um posicionamento crítico a partir da perspectiva de gênero e bioética. A análise foi organizada em três momentos: o contexto do aborto no Chile, no marco dos direitos humanos, as transfoamrações na intimidade e as contribuiçãos da bioética e a perspectiva de gênero ao debabe. Por último, apresentam-se, em forma de reflexão, algumas das tensões existentes, assim como as interrogações para as possíveis pesquisas.

Palavras chave: aborto, debate, direitos humanos, direitos sexuais e reprodutivos, gênero, bioética

* Socióloga, Magíster en Género y Cultura. Investigadora de la Facultad Latinoamericana de Ciencias Sociales FLACSO. Chile Correspondencia: cdides@flacso.cl 


\section{Introducción}

Durante los últimos años hemos asistido a diferentes transformaciones en los ámbitos de la sexualidad y la reproducción en Chile, así como en el resto de los países de la región. Se ha producido un espacio de disputas políticas, científicas, éticas y valóricas, en las cuales es posible visualizar diversas formas de interpretar problemas como el del aborto. En este debate, los discursos biomédicos y bioéticos han alcanzado una legitimidad social de gran relevancia. Es así como la medicina se ha convertido en el un nuevo depositario de la verdad, en el lugar donde expertos, supuestamente objetivos y moralmente neutrales, emiten juicios absolutos $\mathrm{y}$, a menudo, definitivos.

El presente artículo reflexiona sobre el problema del aborto en Chile, a partir de la revisión del contexto histórico actual del país, y plantea una crítica desde la perspectiva de género y bioética. El análisis se ha organizado en tres secciones: el contexto del aborto en Chile en el marco de los derechos humanos, las transformaciones en la intimidad y los aportes de la bioética y la perspectiva de género al debate. Por último, se presentan, a modo de reflexión, algunas de las tensiones existentes así como las interrogantes para posibles investigaciones.

\section{El contexto del aborto en Chile en el marco de los derechos humanos}

En la década de los 90 se constata en Chile una serie de transformaciones en el campo de la vida social y económica del país, cuyas consecuencias se observan en la situación social de las mujeres que comienzan a ocupar espacios que tradicionalmente eran masculinos, como resultado, entre otros factores, de su gradual incorporación al mercado de trabajo y del notorio aumento en los niveles de escolaridad. Por otro lado, nuevos marcos de comprensión de la realidad y, por tanto, del lugar de la mujer en la sociedad empiezan a instalarse. Se incorpora la concepción de las mujeres como sujetos de derecho, la extensión del concepto mismo de "derechos" -y derechos humanos, en particular, hasta alcanzar la precisión de ser derechos humanos de las mujeres-, la introducción de principios de no discriminación, la aspiración a la igualdad y justicia de género, entre los más importantes. Esto como resultado de una combinación de las luchas transnacionales del movimiento feminista y de la acción e influencia de actores institucionales nacionales e internacionales.

La Conferencia Mundial sobre Población y Desarrollo, El Cairo (1994), y la Conferencia Mundial sobre la Mujer, Beijing (1995), han puesto especial énfasis en la relación entre derechos humanos y derechos sexuales y reproductivos, entendidos éstos como el derecho de hombres y mujeres a decidir libremente y tener opciones en lo concerniente a la libertad y seguridad de la persona, sexualidad, derecho a la vida, reproducción, calidad de atención en salud, acceso a la información y otras cuestiones como la privacidad y confidencialidad en la toma de decisiones referidas a la salud sexual y reproductiva, derechos que el Estado tiene la responsabilidad de no transgredir y de adoptar las medidas que sean necesarias para asegurar su ejercicio.

En este marco de transformaciones de los últimos veinte años se han suscitado debates intensos en torno al aborto. En la mayor parte del mundo industrializado el aborto no era catalogado como un delito criminal hasta que, en la mitad del siglo XX, se promulgó una serie de leyes antiaborto, debido, principalmente, a los riesgos que esa intervención tenía entonces para la vida de las mujeres.

En Chile, desde que en septiembre de 1989 se derogara la disposición que por aproximadamente cincuenta años había permitido el aborto 
terapéutico, todos los abortos que se realizan en este país ocurren en la clandestinidad, ya que está penalizado sin excepción(1). En los años previos a 1930, cuando el aborto tuvo el estatus de grave problema de salud pública y se autorizó en caso de grave riesgo para la vida o la salud de la mujer, a través del Código Sanitario, Artículo 119, el aborto inducido había permanecido en el ámbito de la vida privada, y no constituía preocupación de la opinión pública, de las autoridades ni de otras instituciones(2). Con la disminución de la mortalidad infantil -gracias a planes estatales de alimentación y salud y el descubrimiento de los antibióticos- aumentó dramáticamente su número en mujeres desfavorecidas económicamente(3). Cabe señalar que, en ese período de la historia del país, la reflexión sobre el tema se abordó desde la mirada de la salud pública.

Las cifras actuales sobre el número de abortos que se practican son desconocidas, ya que su práctica está penalizada. No obstante, algunos estudios de la última década sobre la incidencia del aborto inducido indican que Chile tiene una de las tasas más altas de América Latina, dado que las estimaciones del número de abortos clandestinos que se practican anualmente en el país varían entre 159.650(4), 200.000(5), y alrededor de 60.000(6) abortos por cada 100 mujeres de 15 a 49 años. Según el informe de The Alan Guttmacher Institute, de 1994, de 451.800 embarazos un 35\% terminó en un aborto inducido(4, p.24).

El diagnóstico realizado por el Instituto Chileno de Medicina Reproductiva (ICMER) y la Corporación de Salud y Políticas Sociales (CORSAPS)(7) da a conocer datos del Programa de Salud de la Mujer del Ministerio de Salud de 2000, donde los egresos hospitalarios por aborto, en los servicios de salud públicos y privados del país, disminuyeron, entre 1990 y 2000, de 36.528 a 29.546, respectivamente. Por su parte, las tasas de egresos por aborto también han mostrado un leve descenso en los últimos años. En 1997 la tasa fue de 115,8 por mil nacidos vivos, de 113,8 en 1998 y de 111,7 en 1999. Estas varían según los servicios de salud del país. En 1999, las tasas más altas observadas fueron en los servicios de salud O'Higgins $(178,8)$ y Atacama $(178,3)$, superando la tasa de egresos por abortos a escala nacional. Esta información es parcial y se refiere a los egresos hospitalarios por complicaciones derivadas de aborto.

La situación descrita determina, según Salazar, diversos riesgos para las mujeres. Por una parte, de orden médico, pues la mayoría de las personas que realiza las intervenciones no tiene competencia técnica, no cuenta con infraestructura y materiales adecuados ni se responsabiliza por las complicaciones derivadas; por otra, de orden psicológico: la imposibilidad de hablar sobre la experiencia del aborto dificulta su elaboración en términos psíquicos, lo que origina diversos síntomas y favorece la repetición de la experiencia(8).

Si bien se constata un descenso de las hospitalizaciones por aborto, esta situación puede ser reflejo de la disminución de los abortos inducidos o, posiblemente, sea el resultado de una mejoría de las técnicas y procedimientos utilizados(9). También es necesario señalar que las tasas de mortalidad por aborto en el país han disminuido drásticamente una vez iniciado el programa de Planificación Familiar en la década de los 60. Esto se constata en la disminución de 10,7 a 0,5 muertes por cada 10.000 nacidos vivos entre 1960 y 2000(7, p.50).

Los estudios más recientes realizados en nuestro país acerca de los procesados por aborto muestran que casi la totalidad de las inculpadas, proveedores y cómplices son mujeres. La mayoría de las abortantes procesadas por este 
delito en Chile son mujeres jóvenes, con hijos, con o sin relación de pareja estable, de baja escolaridad y de escasos recursos(10).

Cabe recordar que en los años setenta(11), a partir del temor al crecimiento demográfico con sus eventuales consecuencias políticas y del desarrollo de nuevas tecnologías anticonceptivas, se inició el debate internacional en relación con los derechos de las personas a la información y a los medios para decidir cuándo tener hijos $\mathrm{y}$ cuántos. Los actores más relevantes han sido los agentes y organismos internacionales -preocupados por el crecimiento de la población mundial- y el movimiento de mujeres, que comenzó a promover los derechos de las personas en materia de salud sexual y reproductiva.

Desde el paradigma del control de la natalidad, como procedimiento altruista de protección de la especie, hasta la postura emergente en el sistema de Naciones Unidas, que establece la regulación de la fecundidad como un derecho de las personas; desde la sublimación de la maternidad, como forma de control moral de la sexualidad, hasta la defensa de la autodeterminación absoluta de las mujeres sobre su cuerpo, impulsada por grupos de mujeres, la reproducción humana, su control y sus representaciones constituyen una arena de debate político, religioso y económico. Un denominador común de esta discusión es la tensión que se produce entre la valoración socio-histórica y políticamente condicionada de la maternidad, y el carácter subjetivo de un proceso que ocurre -en concreto- en el espacio íntimo-sexual $y$, en última instancia, en el cuerpo humano femenino.

Hoy se reconoce que los derechos sexuales y reproductivos son parte de los derechos humanos, como han establecido las conferencias mencionadas. En estas reuniones se diseñó una plataforma de acción que aseguraría el ejercicio de estos derechos para la población, ya que, a pesar de que los acuerdos internacionales no tienen carácter vinculante, sirven para que los Estados formulen las bases de las políticas públicas y fijen acciones tendientes a alcanzar los objetivos internacionalmente acordados. El gobierno chileno adhirió a las definiciones y los programas de acción de estas conferencias.

Otro instrumento jurídico internacional es la Convención sobre la Eliminación de las Todas las Formas de Discriminación contra la Mujer (CEDAW), adoptada por Chile en 1989. Los compromisos adquiridos por el Estado desde esta convención tienen un carácter de obligatoriedad, lo que representa un gran avance en orden a configurar un estatuto sobre derechos sexuales y reproductivos, inspirador de legislaciones locales. Aunque el protocolo facultativo de la CEDAW fue suscrito en 1999, aún está pendiente su ratificación por el Congreso chileno. El país no tiene un cuerpo legal que trate directamente los derechos sexuales y reproductivos, y la normativa existente se refiere a la defensa de los derechos del que está por nacer, la penalización del aborto y protección de la maternidad.

\section{Las transformaciones en la intimidad: sexualidad y reproducción}

Las transformaciones en los ámbitos de la estructura productiva y los procesos de modernización han generado importantes cambios en las pautas culturales y las estructuras sociales, lo que ha influido también en el ámbito de la intimidad de las personas y ha impactado de manera relevante el campo de la sexualidad y la reproducción, es decir, la manera cómo se vive, los comportamientos sexuales y las prácticas discursivas. Según Beck, Giddens y Lash, en la modernidad ya no existirían patrones definidos, más bien habría una reinterpretación de los sentidos de lo sexual, pues ya no hay una coherencia y homogeneidad, sino que el sentido se iría construyendo cotidianamente $(12,13)$. 
La intimidad ha implicado una democratización del "dominio interpersonal" en una forma homologable a la democracia en la esfera pública, lo que ha influido sobre las diferentes instituciones modernas. La vida personal de las mujeres ha entrado en un campo de negociación de la intimidad: se ha convertido en un proyecto personal abierto que ha creado nuevas demandas. Es un proceso donde las mujeres han ejercido un rol importante para develar lo difuso de la dicotomía privado-público. Esto es posible visualizarlo a través de los derechos sexuales y reproductivos como un espacio de ejercicio, a partir de las nuevas concepciones sobre la sexualidad, las tecnologías en salud reproductiva, el aborto, los debates sobre población y desarrollo y la discusión acerca de lo público y lo privado(14).

Según Beck, uno de los aspectos centrales en este cambio en la intimidad ha sido la "autonomía”. Más allá de las discusiones ético-filosóficas respecto del concepto, se trata de rescatarlo como uno de los hilos conductores y componente sustantivo en el escenario de la vida personal, como condición para relacionarse con los otros de forma igualitaria, permitiendo el respeto por las capacidades de los demás, que es nuclear al orden democrático(15).

Los aportes de las investigaciones que se han enmarcado en la perspectiva de género han sido la deconstrucción de la sexualidad y la reproducción, la recuperación del concepto de "sujeto autónomo" y la discusión sobre la frontera entre lo público y lo privado.

Palma señala que, en las últimas décadas, han sido sometidas a crítica en el campo científico las perspectivas teóricas y metodológicas que provienen de las ciencias biomédicas -la epidemiología, la salud pública, la psiquiatría-y las ciencias sociales. La expansión de la epidemia del VIH/SIDA puso en evidencia los límites conceptuales y metodológicos de la investigación del comportamiento sexual humano en los diversos campos de la investigación científica. Este proceso implicó una revisión crítica de la tradición teórica y metodológica, nuevos debates, desarrollos teóricos, deconstrucción de las categorías y clasificaciones, y ampliación de los enfoques hacia las aproximaciones cualitativas y etnográficas(16).

De acuerdo con Weeks, la construcción social de la sexualidad se relaciona con las maneras múltiples e intrincadas en que las emociones, deseos y relaciones son configuradas por las sociedades. Cada sociedad organiza el potencial erótico humano y posee una determinada concepción sobre la sexualidad, la que condiciona normalmente una parte de la conducta y comportamiento de sus individuos, en particular en la participación en las relaciones sexuales, la constitución de una familia -monógama o polígama-, la fecundidad, el comportamiento reproductivo, etc.; es decir, lo permitido, lo prohibido, lo deseable y lo real. Por tanto, la sexualidad es un complejo sociocultural históricamente determinado, consistente en relaciones sociales, instituciones sociales y políticas, así como concepciones del mundo que definen las identidades básicas de los sujetos. Contiene y supone también los roles sociales, las funciones y las actividades económicas asignadas - sobre la base del sexo- a los grupos sociales y al trabajo, al erotismo, al arte, a la política y a todas las experiencias humanas. Consiste, además, en el acceso y la posesión de saberes, lenguajes, conocimientos y creencias específicas, implicando rangos y posiciones con relación al poder(17).

Desde diversas instituciones sociales se tejen redes "morales", "jurídicas" y "biomédicas" que controlan el cuerpo y la sexualidad de las mujeres. Uno de los soportes de estas redes son los discursos hegemónicos sobre aborto que 
circulan en nuestra sociedad. El abordaje crítico de estos discursos deconstruye precisamente el predominio del control público por sobre la subjetividad de las mujeres y su capacidad de autodeterminación, intervenida por la ciencia médica, la religión y el Estado.

La reproducción -y la sexualidad siempre latente como fondo del problema- es también un ámbito discursivo y tiene particulares resonancias para la conservación de un sistema cultural. En toda Latinoamérica la recurrencia argumentativa de sectores conservadores, en cuanto a los roles, normas y valoraciones asignables a las mujeres en razón de su capacidad reproductiva, da una medida de la importancia política que se le asigna. Sin embargo, también es cierto que, en el momento actual, estas $h a-$ blas deben competir con otras discursividades emergentes -aunque con grandes dificultades de divulgación-que tensionan sentidos validados acríticamente desde hace siglos(18).

Por su parte, las organizaciones que trabajan, desde la sociedad civil, en derechos sexuales y reproductivos de la región latinoamericana ${ }^{1}$ han argumentado las luchas por estos derechos a partir de la reivindicación de la autonomía, también denominada "autodeterminación sexual o reproductiva", planteada desde una lógica individual o privada, ligada a la construcción de la individualidad como sujeto sexuado. Su propuesta reclama el derecho a participar en las decisiones estatales y gubernamentales respecto de la regulación de la sexualidad y la reproducción.

1 Comité de América Latina y el Caribe para la Defensa de los Derechos de la Mujer (CLADEM), 2001. Derechos Sexuales y reproductivos desafios para el feminismo contemporáneo. Esta encuesta se envió a organizaciones de mujeres y activistas en materia de DD.SS.RR. de América Latina y el Caribe. La sistematización se realizó con 170 respuestas. Sistematización realizada por María Isabel Cedano.

\section{Los aportes de la bioética al debate sobre aborto en Chile}

En el problema del aborto está en debate la cuestión sobre el inicio de la vida y el derecho de las personas a decidir sobre su fertilidad. El aborto se lleva a cabo para terminar un embarazo no deseado. Este proceso contiene el tema de la maternidad posible y pone en juego una eventual paternidad, entendidas ambas no sólo como generación biológica, sino como ejercicio de crianza y proyección de relaciones por vida.

Considerando lo anterior, la importancia de las construcciones discursivas en las sociedades en general $-\mathrm{y}$ en particular en la sociedad chilena- radica en que estas ejercen un poder en diferentes niveles: por un lado, en un plano intersubjetivo; por otro, en las relaciones con el mundo social. Por medio de los discursos se expresan las representaciones del mundo, se formulan y reproducen ideologías y, por ende, los grupos ejercen poder sobre los conocimientos y opiniones de las personas, de manera de controlar indirectamente sus prácticas.

En Chile, como en otros países de la región, es posible constatar que los discursos bioéticos se han convertido en actores centrales a la hora de tomar decisiones frente a temas como el aborto, la reproducción asistida, la confidencialidad de la información, el consentimiento informado, los protocolos de investigación, la muerte cerebral, los cuidados paliativos, los trasplantes de órganos, entre otros. Una buena parte de estos discursos son atingentes al ámbito de la salud sexual y reproductiva de las mujeres y no constituyen un todo homogéneo, pues tienen diferentes filiaciones -por ejemplo, católica, laica, etc.-, lo que organiza un espacio de debate.

Si bien la reproducción humana y sus consecuencias constituyen asuntos del ámbito privado, relacionados con la vida afectiva de las personas, 
se convierten en un tema político cuando su ocurrencia hace surgir la necesidad de elaborar políticas públicas. Las diferencias ideológicas se basan en valoraciones distintas que - política, social y culturalmente- se asignan no sólo a la maternidad, sino también, y talvez básicamente, al ejercicio de la sexualidad y de la reproducción. Es decir, un hecho íntimo se transforma en un hecho político cuando se le otorga un valor y una importancia específica en el mantenimiento o en la modificación de un orden social.

En esta perspectiva es innegable la importancia de introducir en el análisis la perspectiva de género, ya que los valores asignados a la sexualidad y a la reproducción se vuelven realidad evidente a partir de un embarazo, y éste sólo es posible en el cuerpo de las mujeres. Ellas se constituyen en el campo directo de la normativa para efectos del aborto y, dada esta situación, se transforman en objetos y sujetos de las políticas públicas.

Existen varias formas de entender la bioética. La noción más aceptada, como plantea Durand(19), la describe como la búsqueda de soluciones a los conflictos de valores originados por el progreso científico y tecnológico en el ámbito de las ciencias de la salud y la vida. Por otra parte, Lolas(20) plantea que la bioética se entiende como el empleo del diálogo para articular y resolver, en lo posible, dilemas causados por las ciencias y las tecnologías.

La bioética se puede plantear también como una nueva práctica reflexiva que tiene por objeto comprender y analizar los múltiples procesos sociales e individuales implicados por la enfermedad, la salud y el desarrollo de la tecnociencia. Desde esta perspectiva, independiente de sus progresos en el campo de la interpretación y de la argumentación interdisciplinaria, no puede convertirse en disciplina ni tampoco ocupar un lugar jerárquico respecto de otras. Debe integrar de forma constante las diferentes prácticas científicas y de la salud, el reconocimiento de la existencia de problemas éticos, la consciencia de que en el tratamiento con la naturaleza, con la vida y con los individuos sufrientes se generan desafíos que ponen en juego las finalidades sociales y culturales compartidas. La experiencia de la bioética se expresa en diferentes ámbitos, individuales y colectivos, y, de manera más precisa, en el campo de lo médico en un sentido amplio, de lo jurídico, de lo político, de lo religioso, entre otros $(21,22)$.

La bioética y los discursos que de ella se desprenden no han estado exentos de aproximarse y producir conocimiento en el campo de la sexualidad y la reproducción. Partimos de la base que estudia temáticas relacionadas con la vida y la muerte(23). Por ejemplo, conviene recordar que la discusión sobre el estatuto del embrión se ha relacionado directamente con la experimentación y la reproducción asistida, en particular con la fecundación in vitro. Dado que en esta discusión participan diferentes sectores de la sociedad, algunos conservadores y otros liberales, es interesante identificar los aspectos más relevantes y sus consecuencias en la temática del aborto.

En la discusión bioética sobre el estatuto del embrión y la noción de persona es posible identificar, al menos, tres posiciones generales:

- La argumentación que privilegia el criterio biológico o el punto de vista de la naturaleza. Existiría persona humana desde el momento de la fecundación, lo que implicaría que todo aborto es un homicidio y un infanticidio; métodos tales como el DIU y la anticoncepción de emergencia se consideran también como abortivos(24). Reconocer la persona humana desde el momento de la concepción implica aceptar la existencia de derechos naturales, el primero de los cuales es el derecho a nacer, planteando que desde la primera célula-donde se encuentra presente el genoma humano- se aplican esos dere- 
chos. Esta es la posición del Vaticano, que utiliza los descubrimientos de la biología y, en particular, de la genética(25).

- La segunda posición interpreta la noción de persona y el estatuto del embrión a partir de la autonomía de la voluntad. La persona, en los hechos, es el adulto, al que se educa y se socializa en la perspectiva de la autonomía de la voluntad. Ésta se reconoce gracias a la racionalidad, que podría subdividirse en una conciencia de sí mismo y en una capacidad de comunicación. El embrión, por tanto, no puede tener derechos. Los contenidos que definen y promueven el respeto a la persona no serían válidos para el embrión.

- Una tercera posición filosófica ha intentado desarrollarse en función de las dos anteriores. Se trata de no negar al embrión un estatuto personal, pero tampoco conferir a la célula la misma dignidad que se reconoce a las personas. Esta posición intermedia permite diferenciar las obligaciones morales respecto del cigoto, del embrión implantándose y del feto de 24 semanas. El embrión humano se considera como un sujeto en potencia respecto del cual no es posible actuar sin límites y sin dignidad.

La bioética no ha abordado de manera privilegiada la temática del aborto, aunque sus discusiones a propósito del estatuto del embrión han transformado el contexto en el cual se situaba hace algunos años este problema.

En el debate público sobre el aborto -muchas veces a propósito de otras temáticas como la anticoncepción de emergencia ${ }^{2}$ - existe una asimetría de valoración de los discursos según

2 Dides, C. El Discurso Conservador en el Debate Público sobre Anticoncepción de Emergencia en Chile: Análisis a partir de la prensa escrita (2001-2005). Tesis para optar al grado de Magíster en Estudios de Género y Cultura. Mención Humanidades. Facultad de Filosofía y Humanidades, Universidad de Chile, Santiago, 2005. Por publicar en FLACSO. su proveniencia. Así, el que surge del campo biomédico es un discurso legitimado como saber específico; todavía más, algunos de sus representantes defienden para sí el derecho exclusivo de fundar una política pública y una legislación sobre el aborto.

La producción discursiva en el campo de la bioética, en particular sobre este tema, requiere de un análisis desde la perspectiva de género, porque ello permite indagar las relaciones de poder en los espacios públicos y privados, $\mathrm{y}$ también en los dispositivos culturales -de orden material y simbólico- que reproducen el orden discriminatorio. El interés por dilucidar los procedimientos de reproducción del sistema de género potencia una vocación más expresiva en pos del cambio cultural y la superación de la discriminación de las mujeres, asumido el supuesto que ello contribuye a una democratización de las relaciones sociales, lo que beneficia a la sociedad en su conjunto.

\section{A modo de reflexión}

En Chile existen diferentes dominios discursivos que son parte del debate sobre el aborto. Durante mucho tiempo la institución de la medicina ha constituido un espacio de resolución de los problemas planteados por la sexualidad en general, incluido el aborto. Independientemente de que los discursos biomédicos no fueran similares a los de las organizaciones y movimientos de mujeres, la interpretación del aborto como un problema de salud pública permitió solucionar angustias y necesidades de aquellas con embarazos no deseados, como sucedió en la década de los 60 .

En este contexto - descrito como un espacio de debate ideológico, político, cultural, ético y valórico- es necesario revisar los elementos culturales e ideológicos contenidos en los discursos bioéticos y que son determinantes en la formación de la opinión pública. Es imprescindible, 
además, explorar en los sustratos éticos de los discursos con que los distintos actores se refieren en la última década a los derechos reproductivos y a eventuales legislaciones al respecto ${ }^{3}$.

En el actual y reducido debate sobre el aborto, en Chile -como en otros países de la región- se expresan las valoraciones de la sexualidad, la maternidad, la calidad de vida y la capacidad y derecho de autodeterminación de las personas. También se expresan de manera tácita los significados asignados a la muerte $\mathrm{y}$, posiblemente, se revele también el nivel de elaboración de este tema en la sociedad.

La presencia de este marco de comprensión y de acción sobre la realidad social se ha traducido en las modificaciones de las expectativas e ideales asociados con lo femenino, lo que ha influido en nuevas maneras de las mujeres de concebirse a sí mismas y a sus proyectos vitales. Ciertamente, estas transformaciones no podrían entenderse si no se consideran los cambios sociales más generales, resultado de los procesos de globalización y liberalización de los mercados económicos y culturales, así

3 Actualmente existen dos proyectos de ley presentados en el Parlamento. El primero, presentado en junio de 2002 por diputados/as de la Unión Demócrata Independiente (UDI), apunta a cambiar la ubicación actual del delito de aborto entre los delitos contra la moralidad pública y el orden de las familias al capítulo de los delitos contra las personas, lo que conllevaría un significativo aumento de las penas, tanto para las mujeres que se practican o someten a abortos como para los terceros que intervienen; además, este proyecto propone introducir la figura de arrepentimiento eficaz como circunstancia que permitiría no aplicar la sanción penal a la mujer que aborta y, por último, faculta al juez a conmutar la pena privativa de libertad que se le impone por la realización de trabajos determinados en favor de la comunidad, especialmente en organizaciones de tipo "pro vida". El segundo proyecto, presentado en enero de 2003, apunta a reestablecer el aborto terapéutico en el Código Sanitario tal y como se permitiera antes de su derogación por el Gobierno Militar, esto es, "Sólo con fines terapéuticos se podrá interrumpir un embarazo. Para proceder a esta intervención se requerirá la opinión documentada de dos médicos cirujanos". Este se encuentra en la Comisión de Salud y cuenta con el apoyo de diputados/as tanto de la bancada de la Concertación como de la derecha (en particular, del Partido Renovación Nacional). como la concepción de las relaciones sociales y de sujetos: introducción de la idea de derechos, biografías móviles, nuevos valores e ideales sociales, entre otros.

Los límites de las acciones transformadoras de las organizaciones sociales vinculadas a los temas de sexualidad y reproducción deben entenderse en el contexto de los desafíos que les plantea la configuración del objeto sobre o en torno al cual actúan, así como la conformación del campo de pugna política y social.

Desde esta perspectiva, se plantean al menos las siguientes interrogantes:

- ¿Cuáles han sido las consecuencias esperables en términos de transformación de prácticas intersubjetivas o institucionales en los incipientes debates sobre el aborto en Chile?

- ¿Cuáles son los cambios sociales específicos acontecidos en Chile que influyen en la ausencia de un debate abierto y pluralista sobre el aborto?

- ¿Cómo afecta la ausencia de debate y, por tanto, de políticas públicas específicas en la experiencia de la intimidad y en las concepciones sobre sexualidad de las personas?

- ¿Cuáles son las tensiones existentes entre las nuevas posiciones de actores políticos tradicionales que intervienen en este campo (particularmente la Iglesia y el Estado) y los nuevos actores que reconfiguran el ámbito de pugna política (movimientos sociales por la diversidad sexual, movimientos ecuménicos, discursos tecnocientíficos)?

Se plantean una serie de preguntas, especialmente aquellas que apuntan a la dificultad de debatir sobre el aborto en el país, partiendo de la base que Chile es el único país en el mundo que penaliza el aborto en todas sus 
circunstancias y que su discusión es incipiente, dependiente de otros problemas o temáticas asociadas a la sexualidad, lo que hace que muchas veces sea poco rigurosa y para nada respetuosa de los derechos en el marco de una sociedad democrática.

\section{Referencias}

1. Valenzuela A. Algunos antecedentes en torno al estado actual del aborto terapéutico en Chile. Santiago de Chile: Foro Abierto de Salud y Derechos Reproductivos; 1993.

2. Salazar G, Silva AM. Estudio de la opinión pública sobre el aborto inducido y sus matices. Santiago de Chile: Instituto de la Mujer; 1994.

3. Rosselot J, Mardones F. Salud de la familia y paternidad responsable. La experiencia de Chile 19651988. Revista Médica de Chile 1990; 118(3): 330-338.

4. The Alan Guttmacher Institute. Aborto clandestino: una realidad latinoamericana. New York y Washington; 1994.

5. Requena M, (ed.) Aborto inducido en Chile. Santiago de Chile: Ediciones de la Sociedad Chilena de Salud Pública; 1990.

6. Lavín $\mathrm{P}$, et al. Informe preliminar sobre la caracterización de los casos y costos del tratamiento del aborto hospitalizado en Santiago de Chile. Ponencia presentada en el "Encuentro de Investigadores sobre Aborto Inducido en América Latina y el Caribe". Santafé de Bogotá: Universidad Externado de Colombia; 1994.

7. Shiappacasse V, Casas L, Vidal P, et al. Chile. Situación de la salud y los derechos sexuales y reproductivos. Santiago de Chile: SERNAM, Instituto Chileno de Medicina Reproductiva, Corporación de Salud y Políticas Sociales; 2003.

8. Salazar G. Investigación-acción sobre el aborto inducido mediante el apoyo integral a la mujer postabortante. Santiago de Chile: Instituto de la Mujer; 1993.

9. Foro de Salud y Derechos Sexuales y Reproductivos y Red de Salud de las Mujeres Latinoamericanas y del Caribe. Atención Humanizada del Aborto Inseguro en Chile. Monitoreo del Programa de Acción de la "Conferencia Internacional sobre Población y Desarrollo", El Cairo, 1994. Santiago de Chile; octubre, 2005.

10. Casas L. Mujeres procesadas por aborto. Santiago de Chile: Foro Abierto de Salud y Derechos Reproductivos; 1996.

11. Barzelatto J, Calderon MC, Isaacs S, et al. El aborto en Chile. Elementos para el debate. Santiago de Chile: CORSAPS; 1996.

12. Beck U, Giddens A, Lash S. Modernización reflexiva. Política, tradición y estética en el orden social moderno. Madrid: Alianza Universidad; 1997.

13. Giddens A. La transformación de la intimidad. Madrid: Editorial Cátedra; 1992.

14. Dides C. Aproximaciones a los debates públicos sobre sexualidad y reproducción: aprendizajes sobre la introducción de anticoncepción de emergencia en Chile. En: Cáceres C. Frasca T, Pecheny M, et al. (edit.) Ciudadanía Sexual en América Latina: abriendo debate. Lima: Universidad Peruana Cayetano Heredia; 2004. 
15. Beck U. Hijos de la libertad. México: FCE; 1999.

16. Palma I. Estrategias de prevención en salud sexual y reproductiva en jóvenes en América Latina y el Caribe: hacia una nueva síntesis de enfoques. Informe Técnico. Equipo de Apoyo Técnico Fondo de Población de Naciones Unidas para América Latina y el Caribe; enero 2002.

17. Weeks J. Sexualidad. México: Paidós; 1998.

18. Grau O, Delsing R, Brito E, et al. Discurso, Género y Poder. Discursos públicos: Chile 1978-1993. Santiago de Chile: La Morada. Universidad Arcis; 1997.

19. Durand G. La bioética: naturaleza, principios, opciones. Bilbao: Desclée de Brouwer; 1991.

20. Lolas F. Temas de bioética. Santiago de Chile: Editorial Universitaria; 2000.

21. Zorrilla S, et al. Reproducción asistida. Encuentro entre la bioética y el género. Revista Mujer Salud/Red de Salud de las Mujeres Latinoamericanas y el Caribe 1997; (2).

22. Dides C, (comp.) Bioética y tradiciones. El desafio de la tecnociencia. Santiago de Chile: Centro de Investigaciones en Bioética y Salud Pública. Universidad de Santiago; 1997.

23. Dharkin R. El dominio de la vida. Una discusión acerca del aborto, la eutanasia y la libertad individual. Barcelona: Ariel; 1994.

24. Pedrot P. Le statut juridique de l'embryon et du foetus humain en droit comparé. La Semaine Juridique 1991; 65: 26-30.

25. Mejía MC, Pérez G. Concepciones alternativas sobre sexualidad, reproducción, anticoncepción y aborto. En: Ética y salud reproductiva. México: Programa Universitario de Estudios de Género y Programa Universitario de Investigación en Salud/UNAM; 1996. 